\title{
A Cartography of the Ecstatic and Meditative States
}

\section{Roland Fischer}

In this age so concerned with travel in outer as well as inner-space, it is strange that, while we have detailed charts of the moon, we have no cartography of the varieties of human experience. In order to draft a map of inner space, I am ready to be your travel guide and take you on two voyages: one along the perceptionhallucination continuum of increasing ergotropic (1) arousal, which includes creative, psychotic, and ecstatic experiences; and another along the perceptionmeditation continuum of increasing trophotropic (1) arousal, which encompasses the hypoaroused states of Zazen and Yoga samadhi.

Along the perception-hallucination continuum of increasing arousal of the sympathetic nervous system (ergotropic arousal), man--the self-referential system--perceptually-behaviorally (cortically) interprets the change (drug-induced or "natural") in his subcortical activity as creative, psychotic, and ecstatic experiences (2). These states are marked by a gradual turning inward toward a mental dimension at the expense of the physical. The normal state of daily routine, our point of departure, is followed by an aroused, creative state, which can be characterized by an increase in both data content (a description of space) and rate of data processing ["flood of inner sensation' (3), or most intense time (4)]. However, in the next aroused state on the continuum, acute schizophrenic [or rather "hyperphrenic" (5)] state, further increase in data content may not be matched by a corresponding increase in the rate of data processing. While the creative state is conducive to the evolution of novel relations and new meaning, the psychotic "jammed computer" state interferes with the individual's creative interpretation of the activity of his central nervous system (CNS). At the peak of ecstatic rapture, the outside (physical) world "retreats to the fringe of 
consciousness" (ㅁ), and the individual reflects himself in his own "program." One can conceptualize the normal, creative, "hyperphrenic" and ecstatic states along the perception-hallucination continuum as the ledges of a homeostatic step function (7). While the creative person may travel freely between "normal" and creative states, the chronic schizophrenic patient is stranded in the "jammed computer" state. And the talented mystic, of course, does not need to go through every intermediate step to attain ecstasy.

The mutually exclusive relationship between the ergotropic and trophotropic systems (ㅁ) justifies a separate perception-meditation continuum of increasing trophotropic arousal (hypoarousal) that is continuous with, and to the right of, perception-hallucination continuum (Fig. 1).

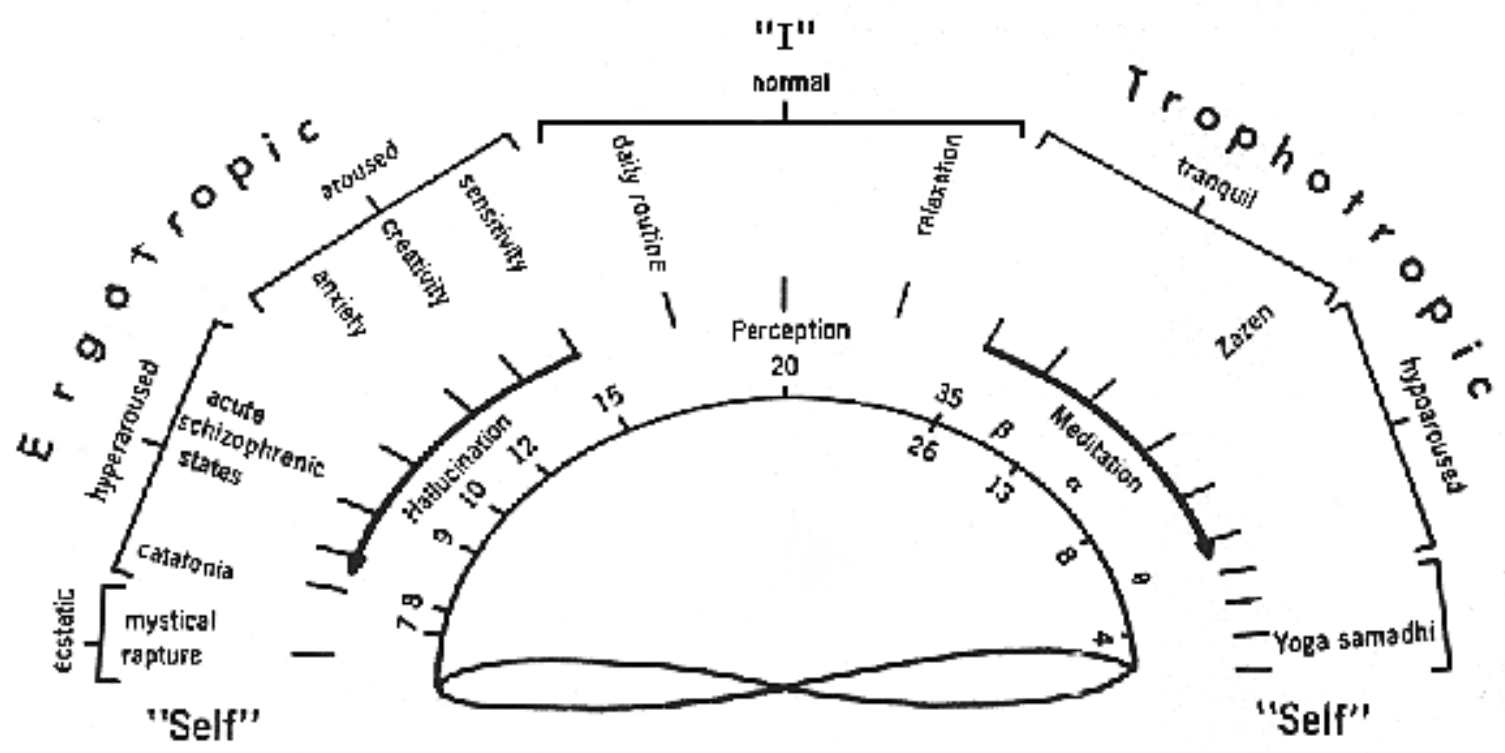

Fig 1. Varieties of conscious states mapped on a perceptionhallucination continuum of increasing ergotropic arousal (left) and a perception-mediation continuum of increasing trophotropic arousal (right). The loop connecting ecstasy and samadhi represents the rebound from ecstasy to samadhi, which is observed in response to intense ergotropic excitation. The numbers 35 to 7 on the perception-hallucination continuum are Goldstein's coefficient of variation (46), specifying the decrease 
in variability of the EEG amplitude with increasing ergotropic arousal. The numbers 26 to 4 on the perception- mediation continuum, on the other hand, refer to those beta, alpha, and theta EEG waves (measured in hertz) that predominate during, but are not specific to, these states (17).

The course of our second trip, therefore, will take us in the opposite direction, along the tranquil perception-meditation continuum, where man may symbolically interpret his gradually increasing trophotropic arousal as Zazen, and, ultimately, samadhi.

That the two continua in Fig. 1 represent two mutually exclusive states of arousal has been well documented by Hess (1) and Gellhom $(\underline{8}, \underline{9})$. The mutual exclusiveness of the ergotropic, and trophotropic systems can also be illustrated by characteristic changes in the frequency of the small, involuntary, micronystagmoid movements of the eye. These rapid scanning movements (with a mean frequency of one per second and an amplitude of 5 to 10 minutes of arc) are regarded as a prerequisite for the fixation of an object in physical space-time (10). The frequency of saccadic movement is increased five to eight fold in response to the ergotropic arousal induced by moderate doses of mescaline, psilocybin, or LSD (D-lysergic acid diethylamide) (10). This increase is also present without drugs in acute schizophrenic (11) [that is, patients in a state of ergotropic arousal, the "alarm reaction" (12) stage of Selye's general adaptation syndrome (13)].

On the other hand, 0.9 gram of alcohol per kilogram of body weight and even sleepiness and fatigue, decreases saccadic frequency (14); more precisely, 0.01 milligram of Valium (diazepam) per kilogram of body weight reduces the saccadic frequency by 9 degrees per second (15). Such a progressive decrease seems to be a characteristic feature of trophotropic arousal along the perception-mediation continuum. That the alpha rhythm appearing on the electroencephalogram (EEG) appears to be phase-locked to the onset of saccades (16) may also be of 
significance, since states of progressively greater trophotropic arousal along the perception-meditation continuum are characterized by EEG waves of progressively lower frequencies (measured in hertz) (17) (see Fig. 1, right). Moreover, since a complete arrest of saccadic frequency, [for example, by optical immobilization of the retinal image (18)] results in periodic fading, disintegration, and fragmented reconstruction of the image, we may postulate that reduced saccadic frequency may be linked with the Yogi's comment that, at the peak of a meditative experience, he can still see "objects," but they have no predicative properties (19).

\section{What An Hallucinations and How Can They Be Measured?}

The hallucinatory or waking-dream states along the perception-hallucination continuum can best be described as experiences of intense sensations that cannot be verified through voluntary motor activity. Note that such a definition does not differentiate between dreams and hallucinations; for example, see the "Three Wise Men" (Fig. 2 unavailable). Two of the three wise men dream with eyes closed, while the third, with eyes open, hallucinates the angel who carries all three away from the "real" world into a mental dimension. We can describe verifiable perceptions, therefore, by assigning to them low sensory-to-motor $(\mathrm{S} / \mathrm{M})$ ratios (20), while non-verifiable hallucinations and dreams can be characterized by increasing S/M ratios as one moves along the perceptionhallucination or perception-hallucination continuum toward ecstasy or samadhi, the two most hallucinatory states (21) (Fig. 1, left and right, respectively). Moderate doses of the hallucinogenic drugs LSD, psilocybin, and mescaline (22) can get one "moving" along the perception-hallucination continuum, whereas minor tranquilizers and some muscle relaxants may initiate travel along the perception-meditation continuum. If high $\mathrm{S} / \mathrm{M}$ ratios do, indeed, reflect hallucinatory experiences, as my definition of hallucinations would imply, it would be important to quantify S/M ratio as a measure of hallucinatory intensity. In fact, a quantitative meaning has been given to the $\mathrm{S} / \mathrm{M}$ ratio by measuring the 
components of a psychomotor performance, specifically, handwriting area and handwriting pressure (20), in volunteers during a psilocybin induced wakingdream state. The techniques for measuring handwriting area (S) (in square centimeters), as well as for obtaining handwriting pressure (M) (in 104dynes averaged over time), with an indicator that operates on a pressure-voltage- tofrequency basis, have been described elsewhere (20). Using these two parameters prior to (T1) and at the peak,(T2) of a psilocybin-induced experience [160 to 250 micrograms of psilocybin per kilogram of body weight], we found in a sample of 47 college-age volunteers a 31 percent (T1 -T2) increase in mean S/M ratio. I should note that the standard deviation on handwriting area at T1 is significantly related to the S/M at T1 $(r=0.4888, P<.01, N=47)$ and that the standard deviation is a simple and useful indicator of the ensuing drug induced increase in S/M ratio ( $r=0.372, \mathrm{P}<.01, \mathrm{~N}=47)$. Moreover, subjects with a large standard deviation on handwriting area at T1 (that is, "variable" subjects), tend to be "perceivers," whereas volunteers with a small standard deviation at T1 ("stable" subjects) tend to be "judgers," in terms of the Myers-Briggs Type Indicator. This self-reporting, Jungian-type personality indicator yields simple, continuous scores on four dichotomous scales: extroversion-introversion, sensation-intuition, thinking-feeling, and judging-perceiving (23). The perceivers also overestimate or contract time more than judgers do at the peak of a psilocybin-induced experience (4): this implies that perceivers move faster and farther along the perception-hallucination continuum than do judgers, who apparently require a larger dose for a comparable experience.

\section{Space and Increasing Hyper and Hypoarousal}

We call man's symbolic interpretation of his CNS activity "perception-behavior" and regard creative, "hyperphrenic," and ecstatic states, as well as Zazen and samadhi, as perceptual-behavioral interpretations of ergotropic and trophotropic arousal, respectively. We may now consider some of the perceptual-behavioral changes, or transformations, that gradually develop as the level of arousal 
increases and decreases along each continuum. One of the most conspicuous transformations is that of "constancies" (24), which in the normal state of daily routine form a learned structure of primary ordering of space and time "out there." Although the newborn infant's only reality, in the beginning, is his CNS activity, he soon learns, by bumping into things, to erect a corresponding model "out there." Ultimately, his forgetting that his CNS activity had been the only reality will be taken by society as proof of his maturity, and he will be ready to conduct his life "out there" in (container) space and (chronological) time (4). This gradually learned and projected model, then, is the representation of a world ordered and stabilized by self-programmed invariances. The adult interprets his CNS activity within this structure of similarity criteria, or "constancies," and thus experience can be said to consist of two processes: the programmed (subcortical) CNS activity; and the symbolic or perceptual-behavioral (cortical) interpretation, or metaprograms, of the CNS activity. I have studied the transformation of certain constancies along the perception-hallucination continuum and find, for example, that the ability to readapt to optically induced spatial distortions, or to maintain the constancy of the visual world, gradually diminishes as a subject turns inward under the influence of psilocybin (25). Another finding revealed that the preferred level of (the constancy of) brightness increases under the influence of hallucinogenic drugs (26), but only in "variable" subjects $(\underline{20}, \underline{27})$--that is, those subjects whose large standard deviations on a variety of perceptual and behavioral tasks indicate a large and varied interpretive repertoire. However, in "stable" subjects, who are characterized by small standard deviations and, thus, by smaller and more predictable interpretive repertoires, the level of preferred brightness decreases when they are under the influence of hallucinogenic drugs. In addition, nearby visual space was found to gradually close in as subjects moved along the perception-hallucination continuum under the influence of moderate doses of psilocybin. This contraction of nearby visual space was observed with two different techniques: monitoring the apparent fronto-parallel plane (28), and handwriting measurements (29). 
The transformation of constancies under ergotropic arousal--specifically as manifested in the psilocybin-induced contraction of nearby visual space--can also be observed in acute schizophrenics under "natural" ergotropic arousal (that is, without hallucinogenic drugs). The transformation of constancies during acute psychotic episodes apparently gives rise to a "vertical displacement of the visual angle," which is implicit in a contraction of visual space and which results in an elevation of the horizon ( $\underline{30})$. Rennert (으), who for years has studied the angle of perspective in the drawings of schizophrenic patients, finds the acuteness of a schizophrenic episode to be significantly related to the height of the horizon in the patient's drawings. In fact, using a ruler, Rennert can predict remission or relapse from the position of the horizon in a drawing: the more severe the schizophrenic episode, the higher the position of the horizon--ultimately, it may even disappear. At the same time (see cover - unavailable), a map-like perspective, or bird's-eye view, of the landscape results, with houses and other significant figures appearing in the foreground. The cover etching, made during an acute (hyperaroused) schizophrenic episode, demonstrates the contraction of nearby visual space, resulting in a raised horizon. Note that the elevation of the horizon forces the animals to walk at a steep angle. Compare with the low horizon in another etching by the same patient after remission (31).

I have also observed that the just noticeable difference (JND) in taste, expressed as Weber fraction, becomes smaller with increasing ergotropic arousal: subjects need fewer molecules of a sapid substance (such as sucrose, quinine, and so on) to taste a JND in sweetness or bitterness. On the other hand, under the influence of tranquilizers of the phenothiazine type, the Weber fraction becomes larger: more molecules are needed to taste a JND (르). Since the Weber fraction is constant at levels of arousal associated with daily routine (within the customary middle range of taste sensitivity) (33), I interpret the above as examples of arousal-induced transformations of constancies. Because the number of molecules necessary to elicit the sensation of a JND gradually decreases during a voyage from the physical to the mental dimension along the 
perception-hallucination continuum, it might be extrapolated that no sapid molecules at all are needed for the experience of taste during ecstasy, the most hyperaroused hallucinatory state.

It should be emphasized that the projection of our CNS activity as location in the physical dimension of space and time "out there" was learned at, and is hence bound to, the lower levels of arousal characteristic of our daily survival routines. That this projection is gradually learned can be supported by Bender's observation that schizophrenic children "do not experience hallucinations of the projected type like adults, but only of the introjected type. They hear voices inside their head or other parts of the body, feel that they originate inside themselves and do not feel persecuted by them" (34).

The constancy of corporeal awareness also undergoes transformations as one moves along the perception-hallucination continuum. For instance, phantom sensations [that is, readaptation phenomena compensating for and correcting distortions of corporeal awareness in physical space-time (35)] gradually diminish and disappear as one moves into the mental dimension under the influence of hallucinogenic drugs (36). Depersonalization phenomena, on the other hand, manifest themselves as changes in body image, and usually accompany the dissolution of ego boundaries during creative, psychotic, ecstatic, or meditative states--whether "natural" or drug-induced--as well as while falling asleep. All of this is to say that the constancy of the "I" is interfered with as one moves along the perception-hallucination continuum from the "I" of the physical world to the "Self" of the mental dimension; Analogously, the perception-meditation continuum (Fig. 1, right) also involves a departure from the "I" to the "Self." These two continua can thus be called "I-Self" continua. As will become clear later, the "Self" of ecstasy and the "Self' of samadhi are one and the same "Self."

The further we progress on the perception-hallucination continuum from the normal through the creative, psychotic, and, ultimately to the ecstatic state (Fig. 
1), the more complete is the transformation, or "unlearning," of the constancies of the physical dimension. Input, or outside information in general, is gradually reduced along this continuum. Thus, Saint Teresa of Avila tells us in her autobiography that, at the peak of a mystical experience, "... the soul neither hears nor sees nor feels. While it lasts, none of the senses perceives or knows what is taking place" (6). Space, then, which was gradually established in everwidening circles during childhood, gradually contracts with increasing arousal and ultimately disappears.

\section{Time and Increasing Hyper- and Hypoarousal}

A gradual contraction and ultimate disappearance is also the rate of chronological time in the physical dimension (of the "l" state) as one progresses along the perception-hallucination or the perception-mediation continuum. In particular, we find that LSD (37) and psilocybin (4) cause, an overestimation of time, the magnitude of which is related to a subject's variability on a perceptual or behavioral test before ingesting the given drug. The greater a subject's variability before ingesting a drug--for example, the retest-variance on his quinine tastethreshold or the standard deviation on his handwriting area--the greater will be his contraction or overestimation of time at drug peak [that is, 150 minutes after the oral administration of 160 to 200 micrograms of psilocybin per kilogram of body weight, when 63 minutes of chronological time (in geometrically increasing intervals) are estimated and recorded (4)]. Moreover, the greater the subject's variability, and thus his contraction of time, the greater will be his "rebound effect" 24 hours after: that is, his underestimation or expansion of time (4). Dividing people, according to the magnitude of their perceptual variability, into "maximizers" ("stable" subjects) and "minimizers" ("variable" subjects) assists one in resolving the hotly debated question of whether time "flies" or "drags" during a hallucinogenic drug-induced experience. Actually, as we have found, it does both: it is overestimated (it "flies" or contracts) by the minimizers, the subjects with a large standard deviation, who prefer to decrease (visual) sensory 
data content and its rate of processing at drug peak; and it is underestimated (it "drags" or expands) by the maximizers, the subjects with a small standard deviation, who prefer to increase data content and its rate of processing at drug peak.

Such contraction of time parallels the already described contraction of nearby visual space. By "time-contraction," I mean an increase in data content within a chronological time span, or, in experiential terms, "the flood of inner sensation" (3); and I imply that, during such an experience, the subject, if without a watch, would arrive early for an appointment. Under the impact of an acute, hallucinogenic drug-induced experience, the subject usually compares the time contraction or increased data content of the mental dimension with his past and present routine performance in physical space-time and has, therefore, to conclude that "time" passes slowly. Note, for instance, Hofmann's classical description of riding home on his bicycle under the influence of LSD: "The trip is about four-miles and I had the feeling of not getting ahead, whereas my escort stated that we were rolling along at a good speed" (38). By comparing the usual rate of revolution of the spokes, as well as the usual rate at which the roadside "passed him by," with his experience of an increased data content or "flood of inner sensation," he had to conclude that he was not getting ahead and that time was dragging. Thus, there is only an apparent contradiction in terms: while experience is shifting from the physical toward the mental dimension, physical or chronological time becomes less and less important. Still, this transitional state can only be described in Aristotelian [dualistic, or two-valued (true-false)] terms of chronological time (39) and by comparing experimental with experiential data. This contradiction resolves itself at the peak of ergotropic or trophotropic arousal, since these purely mental states are timeless and spaceless and in no need of comparative verification.

Increasing Cortical-Subcortical Integration with Increasing Arousal 
If we assume that man, the self-referential system, creates experience through the cortical (that is, perceptual-behavioral) interpretation of his subcortical activity, we may ask about the extent of freedom, or relative independence, of the mind (cortex) from the subcortical substratum. Indeed, man is to a large extent free to interpret his subcortical activity in a variety of ways at levels of arousal associated with daily routine. That this freedom is implicit in the functional independence of the limbic and neocortical systems is dramatically demonstrated by the fact that the electrical discharges resulting from hippocampal seizures are confined to the limbic system (수)

We can find no relation between the extent of psilocybin-induced perceptual and behavioral (or cortical) change and a drug-induced increase in pupillary diameter (41), which is a drug dose-dependent parameter of autonomic activity $(\underline{20}, \underline{42})$. This lack of a relation points to a large degree of freedom of cortical interpretive activity, even under moderate hyperarousal.

The cortical-subcortical independence at the level of daily routine and even moderate levels of arousal is also implicit in the results of Maranon's (43) and Schachter and Singer's (44) experiments, in which the set and setting determined the particular cortical interpretation (from "good trip" to "bad trip") of each subject after his subcortical activity had been altered by an injection of 0.5 cubic centimeter of a 1:1000 solution of epinephrine.

It is now common knowledge that the set and setting, as well as the personality, decisively influence the cortical interpretation of hallucinogenic drug-induced changes in subcortical activity ( $\underline{45})$. What is not fully realized is that set, setting, personality, expectations, and past experiences determine the cortical "effects" of most of the psychoactive drugs when they are used in medically endorsed dosages. In fact, except for the anesthetics and hypnotics, there are no drugs that selectively direct human cognitive (psychological, or, in our terms, cortical) functions. 
With rising levels of ergotropic and trophotropic arousal, however, perceptionbehavior becomes increasingly dependent upon (or less free of) the subcortical substratum that generates it. A cat responds to ergotropic hyperarousal with rage, while at the peak of trophotropic arousal the animal always yawns, curls up, and falls asleep. But man may be compelled to interpret these two extreme states of hyper and hypoarousal as ecstasy and samadhi. This increasing stereotypy (loss of freedom) with increasing ergotropic arousal can be observed, for example, as a decrease in the variability of the EEG amplitude, which Goldstein and others have measured with a Drohocki integrator (46). A decrease in variability is expressed as the coefficient of variation (see the coefficient of variation values for states ranging from relaxation to catatonia in Fig. 1) $(\underline{46}, \underline{47})$. Increasing stereotypy also manifests itself as an increase in the S/M ratio (20), thus indicating an intensification of inner sensations, accompanied by a loss in the ability to verify them through voluntary motor activity. Such high S/M ratios are implicit in the statements uttered during both drug-induced hallucinations and the hallucinations of schizophrenics: "of being hypnotized," "of being not free." "of being overpowered," "of being paralyzed," and so on, and in the mystic's inability to experience the subject-object dichotomy of daily routine in the physical dimension.

Apparently, then, an increase in ergotrophic arousal is paralleled by a restriction in the individual's repertoire of available perceptual-behaviora1 interpretations. This restriction implies that certain levels can only be interpreted as creative (artistic, scientific, religious) or psychotic experiences (48). Although a religious interpretation is a common feature of catatonia (49), ecstasy, which is the mystical experience of the Oneness of everything, results from a creative breakthrough out of catatonic hyperarousal. During the ecstatic state, there is neither capacity nor necessity for motor verification of the intense sensations. In the mental dimension, in contrast to the physical, the all-pervasive experience of absolute certainty does not require further verification (50) and will be structured according to current mythology or the belief system of a St. Francis, Pascal, or 
Ramakrishna. What is one man's loss of freedom, therefore, may be another's gain in creativity.

An increasing stereotypy can also be observed along the perception-meditation continuum of increasing trophotropic arousal (see Fig. 1, right); this enables one to gradually exclude stimulation from without and turn attention inward.

Continuous trains of alpha waves accompany these changes, and the dominant frequency of the alpha pattern decreases toward the alpha-theta border region, until some subjects, in a state of reverie, produce long trains of theta waves (51) [see the beta, alpha; and theta waves ( $\underline{52})$, measured in hertz, in Fig. 1, right]. According to Green et al. (17), the "alert inner-focused state is associated with the production of alpha rhythm;" in this state Zen masters show an alphablocking response to auditory clicks, but, in contrast to normal controls, do not habituate to these stimuli (51). Since the alpha rhythm is not altered or blocked by flashing lights, sounding gongs, or the touch of a hot test tube during the deep meditation of Indian Yoga masters (53), the Yoga samadhi apparently represents a more intense state of trophotropic arousal than Zazen does and must also express a greater inability to function in physical space-time than Zazen does. In fact, a Yoga master denies noticing-any outside stimuli during deep meditation, whereas control subjects show alpha-blocking with as little stimulus as a flashing light (as do the Yoga masters themselves when not meditating).

"Self": The Knower and Image-Maker; and "I": The Known and Imagined We have seen that the departure from the physical dimension during a voyage on the perception-meditation continuum is accompanied by a gradual loss of freedom, which is manifested in the increasing inability to verify the experience through voluntary motor activity (3). At the peak of trophotropic arousal, in samadhi, the meditating subject experiences nothing but his own self-referential nature, void of compelling contents. It is not difficult to see a similarity between the meditative experience of pure self-reference and St. Teresa's description of 
her ecstasy: in both timeless and spaceless experiences the mundane world is virtually excluded. Of course, the converge is true of the mundane state of daily routine, in which the oceanic unity with the universe, in ecstasy and samadhi, is virtually absent. Thus, the mutual exclusiveness of the "normal" and the exalted states, both ecstasy and samadhi, allows us to postulate that man, the selfreferential system, exists on two levels: as "Self" in the mental dimension of exalted states; and as "I" in the objective world, where he is able and willing to change the physical dimension "out there." In fact, the "I" and the "Self" can be postulated on purely logical grounds. See, for instance, Brown's reasoning ( $\underline{54})$ that the universe is apparently ... constructed in order (and thus in such a way as to be able) to see itself. But in order to do so, evidently it must first cut itself up into at least one state which sees, and at least one other state which is seen. In this severed and mutilated condition, whatever it sees is only partially itself... but, in any attempt to see itself as an object, it must, equally undoubtedly, act so as to make itself distinct from, and therefore, false to, itself. In this condition it will always partially elude itself.

In our terminology, the "Self" of exalted states is that which sees and, knows, while the "I" is the interpretation, that which is seen and known in the physical space-time of the world "out there." The mutually exclusive relation between the "seer" and the "seen," or the elusiveness of the "Self" and the "I" may have its physiological basis in the mutual exclusiveness of the ergotropic and trophotropic systems (

A discernible communication between the "Self" and the "I" is only possible during the dreaming and hallucinatory states, whether drug-induced or "natural." These states can be located approximately between coefficients of variation 10 and 13 on the perception-hallucination continuum (Fig. 1, left) and in the 9 to 12 hertz EEG range on the perception-meditation continuum (Fig. 1, right). Such "I""Self" communication is the creative source of art, science, literature, and religion. 
In spite of the mutually exclusive relation between the ergotropic and trophotropic systems, however, there is a phenomenon called "rebound to superactivity," or trophotropic rebound, which occurs in response to intense sympathetic excitation (55), that is, at ecstasy, the peak of ergotropic arousal (56). A rebound into samadhi at this point can be conceived of is a physiological protective mechanism; Gellhorn $(\underline{8,9})$ was among the first to notice that the rebound of the trophotropic system is not confined to the autonomic branches, but also causes significant changes in behavior. Thus, repetitive stimulation of the reticular formation in the midbrain increases the arousal level in awake cats, but this phase is followed by one in which the animal yawns, lies down, and finally falls asleep. This rebound phase is associated with the appearance of theta potentials in the hippocampus (57), just as the corresponding human trophotropic reboundsamadhi-is characterized by theta potentials (17) (see Fig. 1, right). These rebound or reversal phenomena between ecstasy and samadhi $(\underline{8,9})$ are illustrated by the loop (툐) connecting the two extreme exalted states in Fig. 1.

The "Self" of ecstasy and samadhi are one and the same, as if the reflecting surface of a lake in Fig. 1 embraced both exalted states. If the level of water in such a lake were gradually raised, it would intersect successive and corresponding hyper and hypoaroused states. The intersected states represent levels of gradually diminishing subjectivity (less "Self') and increasing objectivity (more "I"), until eventually the objective "I"-state of the world is reached. Thus, each level of water would connect a hyper and hypoaroused state with a specific subjectivity/objectivity (or "Self"-to"I") ratio, implying a similarity between those pairs of hyper and hypoaroused states that are connected by gradually raised levels of water. This similarity might, for example, be used to account for the success of the widely practiced narcoanalytic technique of abreacting a traumatic, hyperaroused experience in a hypoaroused state of similar "Self"-to-"I" ratio. The similarity between corresponding hyper and hypoaroused states could also account for the hyperamnesic phenomena of the hypoaroused elderly, who 
clearly recall the hyperaroused experiences of their youth, but do not recall more recent experiences (모).

During the "l"-state of daily routine, the outside world is experienced as separate from oneself, and this may be a reflection of the greater freedom (that is, separateness or independence) of cortical interpretation from subcortical activity. With increasing ergotropic and trophotropic arousal, however, this separateness gradually disappears, apparently because in the "Self"-state of ecstasy and samadhi, cortical and subcortical activity are indistinguishably integrated. This unity is reflected in the experience of Oneness with everything, a Oneness with the universe that is oneself.

\section{Sign-Symbol-Meaning Transformations}

The separateness of subject and object during the daily routine levels of arousal (in the "I"-state) has been elaborated in our customary, rational, Aristotelian logic and language--a two valued (either-or, true-false) logic that discounts the interaction between observer (subject) and observed (object) (60). This separateness of object and subject, as we have seen, is a reflection of the relative independence of cortical interpretation from subcortical activity and is of survival value in the "l"-state, where the subject must make decisions of life and death by manipulating objects (through voluntary motor activity).

But when we depart along either continuum from the "l" toward the "Self," the separateness of object and subject gradually disappears and their interaction becomes the principal content of the experience. This interaction, again, is a reflection of the gradually increasing integration of cortical and subcortical activity. In this state of Unity, the separateness of subject and object that is implicit in dualistic, Aristotelian logic and language becomes meaningless; only a symbolic logic and language can convey the experience of intense meaning. Apparently, then, meaning is "meaningful" only at that level of arousal at which it is experienced, and every experience has its state-bound meaning. During' the 
"Self"-state of highest levels of hyper or hypoarousal, this meaning can no longer be expressed in dualistic terms, since the experience of unity is born from the integration of interpretive (cortical) and interpreted (subcortical) structures. Since this intense meaning is devoid of specificities, the only way to communicate its intensity is the metaphor; hence, only through the transformation of objective sign into subjective symbol in art, literature, and religion can the increasing integration of cortical and subcortical activity be communicated.

The transformation of sign to symbol is also apparent in the visual realm, where the constancies of space and time are replaced by geometric-ornamental rhythmic structures, the "hallucinatory form constants" of Kluver (61). In the light of my own experience, I would extend Kluver's observations to include hyper and hypoaroused hallucinatory experiences in general, whether electrically (62), "naturally" or drug-induced (63). The hallucinatory constancies are "magical symbols" visible or audible metaphors within a structure of symbolic logic and language, the language of hyper and hypoaroused hallucinatory states, and are at the base of the general tendency toward geometric-rhythmic ornamentalization. For example, both the rose windows of Gothic cathedrals and the mandalas of Tantric religious art (64) are ritualized hallucinatory form constants. The tendency toward ornamentalization, however, is not reserved to visual imagery, but also governs the order of poetic and musical rhythm, imposing an all-pervasive metrum and harmony on the hallucinatory creativereligious states (65); the rhythm of music, poetry, and language corresponds to the geometric ornamental rhythm of the visual realm. Therefore, the manneristic (66) hallucinatory-creative style of art and literature is regarded as a projection and elaboration of the geometric-rhythmic ornamental fabric of hyper and hypoaroused states.

\section{State-Boundaries}


Inasmuch as experience arises from the binding or coupling of a particular, state or level of arousal with a particular symbolic interpretation of that arousal, experience is state-bound; thus, it can be evoked either by inducing ("naturally," hypnotically, or with the aid of drugs) the particular level of arousal, or by presenting some symbol of its interpretation, such as an image, melody, or taste. "Acquired aversions to tastes following illness are commonplace in humans. The knowledge that the illness was caused by the stomach flu and not the Sauce Bernaise does not prevent the sauce from tasting bad in the future" (67). Alcohol induces the state of arousal necessary for the recall of a state-bound experience in the film City Lights. Here, Charlie Chaplin saves a drunken millionaire from attempted suicide, and so becomes his good friend. When sober, however, the millionaire does not remember Charlie. However (68):

...the millionaire does not stay sober long. When he is drunk again, he spots Charlie and treats him like a long-lost friend. He takes Charlie home with him, but in the morning, when he is again sober, he forgets that Charlie is his invited guest and has the butler throw him out.

Evidently, consciousness extends either between states of drunkenness; or between states of sobriety; but there is complete amnesia between the two discontinuous states of sobriety and drunkenness, states with characteristic and different "Self"-to-"I" ratios.

Charlie's story has been recently modeled and scientifically validated by Goodwin et al. (69), who had 48 subjects memorize-nonsense syllables while drunk. When sober, these volunteers had difficulty recalling what they had learned, but they could recall significantly better when they were drunk again. Bustamante et al. (70) also observed amphetamine-induced (20 milligrams) excitatory, and amobarbital-induced (200 milligrams), "inhibitory," state-dependent recall of geometric configurations. His volunteers both memorized and later recalled the configurations under one of the two drugs. I submit, however, that while remembering from one state to another is usually called "state-dependent learning" (71) (implying that the individual was confronted with a learning task), 
extended practice, learning, or conditioning is not necessary for producing "state boundness." On the contrary, a single experience may be sufficient to establish state-boundness.

Deja vu experiences and the so-called LSD flashbacks are, I believe, special cases of the general phenomenon of state-boundness, Note that neither focal lesions nor molecules of a hallucinogenic drug are necessary for the induction of a flashback--a symbol evoking a past drug experience may be sufficient to produce an LSD flashback (프).

An 18-year old boy had a "bum trip" on "acid" and could not "come down" for two weeks. After he drank wine with a group of friends and was told by one of them that the wine contained a high dose of LSD (which it did not), he experienced hallucinations continuously for 14 days.

And here is the story of a"'flashback" involving no drugs whatsoever $(\underline{73})$.

I was in love with a college classmate, but he married someone else. I also married, and even after four years and a beautiful baby I still dreamed about this fellow. Whenever I saw a car like his, my heart would pound even though he had left town years before and I knew it couldn't possibly be his.

It follows from the state-bound nature of experience, and from the fact that amnesia exists between the state-of normal daily experience and all other states of hyper- and hypoarousal, that what is called the "subconscious" is but another name for this amnesia. Therefore, instead of postulating one subconscious, I recognize as many layers of self-awareness as there are levels of arousal and corresponding symbolic interpretations in the individual's interpretive repertoire. The many layers of self-awareness, each with its characteristic "Self"-to-"I" ratio, remind one of the captain with girl friends in many ports, each girl unaware of the 
existence of the others, and each existing only from visit to visit (that is, from state to state). This is how multiple existences become possible: by living from one waking state to another waking state; from one dream to the next; one amobarbital narcoanalysis session to the next (74); from LSD to LSD (다); from epileptic aura to aura (6) $)$; from one creative, artistic, religious, or psychotic inspiration or possession to another creative artistic, religious, or psychotic experience; from trance to trance; and from reverie to reverie.

\section{References and Notes}

1. W. Hess, Das Zwischenhirn und die Regullerung von Kreislauf und Atmung (Thieme, Leipzig, 1938); Das Zwischenhirn (Schwabe, Basel, 1949). Ergotropic arousal denotes behavioral patterns prepatory to positive action and is characterized by increased activity of the sympathetic nervous system and an activated psychic state. These states may be induced either naturally or, for example, through hallucinogenic drugs. Trophotropic arousal results from an integration of parasympathetic with somatomotor activities to produce behavioral patterns that conserve and restore energy, a decrease in sensitivity to external stimuli, and sedation. During ergotropic and trophotropic arousal, "alterations in autonomic activity are not confined to the visceral organs, but induce changes in cortical activity" [W. Hess cited by Gellhorn(包].

2. R. Fisher, in Psychiatry and Art, vol. 2, Art Interpretation and Art Therapy, I. Jakab, Ed. (Karger, Basel, 1969), p. 33.

3. R. Gelpke, quoted by A. Hofmann. In Sonderabdruk aus dem Basler Stadtbuch (Basel 1964).

4. R. Fischer, in Proceedings of the 4th International Congress of Pharmacology (Schwabe, Basel, 1970), vol. 33, p. 28; Ann. N.Y. Acad. Sci. 138, 440 (1967). 
5. The word "hyperphrenic" was suggested to me by Dr. A. Bader, Lausanne, Switzerland.

6. Saint Teresa, The Life of Saint Teresa, J. M. Cohen, Transl. (Penquin, Baltimore, 1957), p. 142.

7. W. Ashby, Design for a Brain (Wiley, NY 1960), p. 88.

8. E. Gellhorn, Psychol. Forsch. 34, 48 (1970).

9. __ J. Nerv. Ment. Dis. 147, 148 (1968).

10. E. Hebbard and R. Fischer, Psychopharmacologia 9, 146 (1966).

11. J. Silverman and K. Gaarder, Percept. Mot. Skills 25, 661 (1967).

12. R. Fischer, J. Nerv. Ment. Dis. 119, 492 (1954).

13. H. Selye, J. Clin. Endocrinol. Metab. 6, 117 (1946).

14. M. C. Franck and W. Kuhlo, Arch. Psychiat. Nervenkr. 213, 238 (1970).

15. J. Aschoff, ibid., 211, 325 (1968).

16. K. Gaarder, R. Koresko, W. Kropff, Electroencephalogr. Clin. Neurophysiol. 21, 564 (1966).

17. E. Green, A. Green, E. Walters, J. Transpersonal Psychol. 1, 1 (1970).

18. R. D. Ditchburn and D. Fender, Opt. Acta 2, 128 (1955). Immobilization is accomplished by attaching a tiny mirror to a contact lens in such a way that the image will follow micronystagmoid movements of the eye. Also see R. Prichard, W. Heron, D. Hebb [Can. J. Psychol. 14, 67 (1960)]. 
19. K. Behanan, Yoga, A Scientific Evaluation (Dover, NY 1937), p. 223. The cobra has fixed eyes to begin with; therefore, to compensate for the lack of scanning eye movements, it must sway its head rhythmically to fixate the image of its victim. (If nothing else, one practical application of this paper may be the following: whenever you meet a cobra, swing along with him and he won't be able to locate you.)

20. R. Fischer, T. Kappeler, P. Wisecup, K. Thatcher, Dis. Nerv. Syst. 31,91 (1970); K. Thatcher, T. Kappeler, P. Wisecup, R. Fischer, ibid., p. 181.

21. R. Fischer, ibid, 30, 161 (1969). J. Strauss's evidence also suppors this concept of the continuous, nondiscrete nature of perceptual and hallucinatory experience [Arch. Gen. Psychiat. 21, 581 (1969)]. Our definition of hallucinations or dreams as experiences characterized by a high $S / M$ ratio is free of value judgment, thus implying that hallucinatory experience can be labeled pathological, artistic, religious, and so on, according to one's taste [and taste threshold; see R. Fischer, in Gustation and Olfaction, G. Ohloff and A. E. Thomas, Eds. (Academic Press, NY 1971), pp. 187-237].

22. The cross-tolerance between LSD, psilocybin or mescaline [H. Isbell, A. Wolbach, A. Wikler, E. Miner, Psychopharmacologia 2, 147 (1961); A. Wolbach, H. Isbell, E. Minor, ibid, 3, 1 (1962)] as well as the characteristic square-wave pattern of saccadic movement they elicit [E. Hebbard and R. Fischer, ibid., 9, 146 (1966)] mark these drugs as the hallucinogenic, psychotomimetic, psychedelic, or psychodysleptic drugs. It is implied, therefore, that any state which can be induced by one of these drugs can be duplicated by the others as well.

23. R. Corlis, G. Splaver, P. Wisecup, R. Fischer, Nature 216, 91 (1967).

24. "Constancies" assure the recognition of identity; they refer to what Piaget meant by the "conservation" of area, distance, length, volume, and so on [J. 
Plaget, B. Inhelder, A. Szeminska, The Child's Conception of Geometry, E. A. Luzer, Transl. (Routledpge, London, 1960), p. 390]. For example, wearing prism spectacles results in a variety of visual distortions which, due to the cortical transformation of subcortical (retinal) information, gradually disappear with time. Perceptual-behavioral constancies can be formalized as information (or signal to noise) ratios; that is, as dimensionless quantities [R. Fischer, F. Griffin, R. C. Archer, S. C. Zinsmeister, P. S. Jastram, Nature 207; 1049 (1965)].

25. R. Hill, R. Fischer, D. Warshay, Experientia 25, 171 (1969).

26. R. Fischer, R. Hill, D. Warshay; ibid., p. 166. (1969).

27. R. Fischer, P. Marks, R. Hill, M. Rockey, Nature 218, 296 (1968); R. Fischer, In Origin and Mechanisms of Hallucinations, W. Keup, Ed. (Plenum, New York, 1970) pp. 303-332.

28. R.. Fischer, R. Hill, K. Thatcher, J. Schelb, Agents Actions 1, 190 (1970).

29. R. Hill and R. Fischer, Pharmokopsychiat. Neuro-Psychopharmakol. 3, 256 (1970).

30. H. Rennert, Confin. Psychiat. 12, 23 (1969).

31. L. Navratil, ibid., p. 30.

32.R. Fischer and R. Kaelbling. in Recent Advances In Biological Psychiatry, J. Wortis, .Ed. (Plenum, New York, 1967), vol. 9, p. 183; R. Fischer, L. Ristine, P. Wisecup, Biol. Psychiat. 1, 209 (1970).

33. R. Fischer, H. Dunbar, A. Sollberger, Arnzneimittel-forschungs 21, 135 (1971).

34. L. Bender, in Psychotomemtic Drugs, D. Elron, Ed. (Raven, New York, 1969), p. 267. 
35. R. Fischer, Perspect. Biol. Med. 12, 259 (1969). The loss of a limb or an organ, for instance, can be regarded as a distortion of corporeal awareness; the phenomenon that corrects for this distortion results in a very real feeling that the lost limb or organ is still there, but this is only a phantom sensation.

36. S. Kuromaru, S. Okada, M. Hanada, Y. Kasahara. K. Sakamoto, Psychiat Neurol. Jap. 64, 604 (1962).

37. R. Fischer, Ann. N.Y. Acad. Sci. 96, 44 (1962).

38. A. Hofmann, in Discoveries In Biological Psychiatry, F. Ayd and Blackwell, Eds. (Lippincott, Philadelphia, 1970), chap. 7.

39. R. Fischer, in The Voices Of Time, J. Fraser, Ed. (Braziller, New York, 1966), p. 357.

40. P. MacLean, J. Neurosurg. 11, 29 (1954); Amer. J. Med. 25, 611 (1958).

41.The mean increase in pupillary diameter induced by 160 micrograms of psilocybin per kilogram of body weight in 34 college-age male volunteers was 0.93 millimeter, standard deviation $= \pm 0.52$; whereas in 13 females, the mean increase was 0.56 millimeter, standard deviation $= \pm 0.27$.

42. R. Fischer and D. Warshay. Pharmakopsychiat. Neuro-Psychopharmakol. 1, 291 (1968); R. Fischer, Perspect. Biol. Med. 12; 259 (1969).

43. G. Maranon, Rev. Fr. Endochrol. 2, 301 (1924).

44. S. Schachter and J. Singer, Psychol. Rev. 69, 379 (1962).

45. H. Lennard, L. Epstein. A. Bernstein, D. Ransom, Science 169, 438 (1970).

46. L. Goldstein, I. Murphree, A. Sugerman. C. Pfeiffer, E. Jenney, Clin. Pharmacol Ther. 4, 10 (1963). 
47. K. Thatcher, W. Wiederholt, R. Fischer, Agents Actions 2, 21 (1971); G. Marjerrison, A. Krause, R. Keogh, Electroencephalogr. Clin. Neurophysiol. 24, 35 (1967).

48. R. Fischer, in Genetic Factors in "Schizophrenia, "A. Kapan, Ed. (Thomas, Springfield, IL, in press).

49. B. Pauleikhoff, Fortschr. Neurol. Psychiat. 37, 476 (1969). The distinction between a conversion experience is pointed out by $\mathrm{H}$. Weibrecht [Beitrage zur Religionspsychopathologie, insbesondere zur Psychopathologie der Bekehrun (Scherrer, Heidelberg, 1948)] His four schizoprhenics experienced a religious conversion during their psychoses. Although after remission they had insight into the diseased nature of their psychoses, they continued to regard their conversion experiences as valid turning points. We conceptualize conversion experiences as confrontations and dialogue between a man's worldly "l" and his ecstatic (or meditative) "Self," resulting in the creation of a more consistent personality--one that has "found its style." [R. Fischer, Confin. Psychiat. 13, 1 (1970); ibid., in press].

50. Pascal recorded at the peak of his decisive religious illumination: "Fire. God of Abraham, God of Issac, God of Jacob, not of the philosophers and the scientists. Certainty. Certainty." [M. Arland, Pascal(I'Enfant Poete, Paris, 1946) pp. 120-121].

51. A. Kasamatus and T. Hirai, Psychologia 6, 89 (1963); Folia Psychiat. Neuro. Jap. 20, 315 (1966).

52. It is likely that these parietal, low-frequency EEC wave are related to dendritic field-potential charges. 
53. B. Anand, G. Chhina, B. Singh, Electroencephalogr. Clin. Neurophysiol. 13, 452 (1961).

54. G. Brown, Laws of Form (Allen, London, 1969), p. 105. Saint Teresa elegantly expressed this partial elusiveness: "There remains the power of seeing and hearing: but is as if the things heard and seen were all a great distance far away" [in E. Underhill, Mysticism (Methuen, London, 1912), p. 450].

55. E. Gellhorn, Acta Neuroveg. 20, 181 (1959).

56. Such rebound would be called "inhibition" in pavlovian terminology [I. Pavlov, in C. Murchison, Psychologies of 1930 (Clark Univ. Press, Wocester, MA, 1930), p. 213], and "phase of resistance" by Selye [Stress Acta, Montreal, 1950), pp. 1543]. It can also be accounted for within the frame of Wilder's Law of Initial Value, which states that "the higher the initial value of a measured function, the lower is the tendency of the system to respond to furthering stimuli, while the highest as well as the lowest values tend to result in a reversal of action (italics mine)" [J. Wilder, J. Psychother. 12, 199 (1958)].

57. P. Parmeggiani, Brain Res. 7, 350 (1968).

58. The "loop" in Fig. 1 has also been independently suggested to me (on experiential grounds alone) by both Dr. J. H. M. Whiteman, University of Cape Town, South Africa, and Marilyn Delphinium Rutgers, Glen-Ellen, CA. I am gratefully indebted to them, as well as to Primarius Dr. Leo Navratil, Gugging, Austria, for sharing with me their intuition and scholarship.

59. This example was suggested to me by Primarius Dr. Leo Navratil, Gugging, Austria.

60. For example, neither quinine molecules nor a subject's taste receptors are bitter per se--bitterness results only during interaction of the two. Therefore, no 
taster, no bitterness (just as there can be no image or sound of a falling tree without a viewer or listener). [See R. Fischer, Dis. Nerv. Syst. 30, 161 (1969)].

61. H. Kluver, Mescal and Mechanism of Hallucinations (Phoenix, Univ. of Chicago Press, Chicago, 1966). p. 66.

62. M. Knoll, J. Kugler, D. Hofer, S. Lawder, Confi. Neurol. 23, 201 (1963).

63. W. Keup, Ed., Origin and Mechanisms of Hallucinations (Plenum, New York, 1970),

pp. 95-210.

64. A. Mookerjee, in Tantra-Kunst, R. Kumar, Ed. (Basillius, Basel, 1967-1968), p. 11.

65. R. Fischer, Confi. Psychiat. 13, 1 (1970).

66. See L. Navratil, Schizophrenie und Kunst (Deutsches Taschenbuch Verlag, Munchen, 1965), p. 35; Schizophrenie und Sprache (Deutsches Taschenbuch, Munchen, 1966), p. 162.

67. M. Seligman, Psychol. Rev. 77, 416 (1970).

68. G. McDonald, M. Conway, M. Ricci, Eds. The Films of Charlie Chaplin (Citadel, NY, 1965), p. 191.

69. D. Goodwin, B. Powell, D. Bremer, H. Holne, J. Stern, Science 163, 1358 (1969).

70. J. Bustamante, A. Jordan, M. vila, A. Gonzalez, A. Insus, Physiol. Behav. 5, 793 (1970).

71. R. Fischer, Dis. Nerv. Syst. 32, 373 (1971);___ and G. M. Landon, Brit. J. Psychiat., in press. The later paper also includes an extensive discussion of 
state-dependent learning in human beings and animals. Note that this term could just as well be "state-dependent adaptation," since learning and adaptation are as indistinguishable from each other as hallucinations and dreams.

72. L. Tec, J. Amer. Med. Ass. 215, 980 (1971).

73. From "Dear Abby," in Citizen Journal, Columbus, Ohio, 13 Jan 1971, p. 21.

74. Primarius Dr. Raoul Schindler, Vienna, informed me at Linz in 1969 that a patient's thread of thought in narcoanalyis resumes, after an injection of amobarbital, exactly where it left off at the end of the previous session.

75. Dr. Hanscarl Leuner, Gottingen, Germany, also confirms (1970) that his patients in LSD-supplemented or psycholytic therapy regard each LSD experience as a continuation of the last.

76. M. Horowitz, J. Adams and B. Rutkin [Arch. Gen Psychiat. 19, 469 (1968)] report from the case history of an epileptic girl that the imagery of every aura consisted of "pigs walking upright like people." In early grade school the girl would rip open her blouse during a seizure, but, of course, she would not remember this after the seizure. Nevertheless, her classmates called her a pig, and it was this pig which she saw "walking upright" in each aura.

77. The author is professor of experimental psychiatry and associate professor of pharmacology at the Ohio State University College of Medicine, Columbus 43210. This article is adapted from a paper presented at the AAAS symposium on "Mood, Behavior, and Drugs" in Chicago, IL, 28 December 1970, and from a seminar given on 20 January 1971 at the department of psychiatry, Johns Hopkins University, Baltimore, MA.

Supported in part by National Institute of Mental Health grant 1 RO3 MH1763301 and general research support grants. I am indebted to Sandoz Pharmaceuticals, Basel, Switzerland, and to Dr. John A. Scigliano, former 
executive secretary, FDA-PHS Psychotomimetic Agents Advisory Committee, Bethesda, Maryland, for generously providing me with psilocybin (under IND3350). I am also grateful to Dr. Peter Gwyane, James Scheib, and Pamela Furney for their devoted competence and to my creative artist wife, Trudy, for vital perceptual as well as cognitive illumination. 